\title{
德国小蠊性信息素类似物的设计与合成
}

\author{
王晓波 ${ }^{a} \quad$ 张钟宁 $b$ 杨新玲 ${ }^{*}, a$ \\ ( ${ }^{a}$ 中国农业大学理学院应用化学系 北京 100193) \\ $\left(^{b}\right.$ 中国科学院动物研究所农业虫害鼠害综合治理研究国家重点实验室＼cjkstart北京 100101)
}

\begin{abstract}
摘要 德国小蠊是世界上最难治理的卫生害虫之一. 昆虫性信息素可作为一种安全有效的引诱剂用于害虫的综合治 理. 德国小蠊性信息素(blattellaquinone)因其结构不稳定而妨碍了它的实际应用. 为了发现结构新颖且稳定的性信息素 类似物, 对 blattellaquinone 的结构中苯醌 $(\mathbf{A})$ 、酯基 $(\mathbf{B})$ 和脂肪链 $(\mathbf{C})$ 部分进行了改造, 设计并合成了一系列类似物, 结构 均通过 ${ }^{1} \mathrm{H} N M R$, IR 和 HRMS 分析确证.
\end{abstract}

关键词＼cjkstart德国小蠊; 小蜬醌; 性信息素类似物; 合成

\section{Design and Synthesis of German Cockroach (Blattella germanica) Sex Pheromone Analogues}

\author{
Wang, Xiaobo $^{a} \quad$ Zhang, Zhongning ${ }^{b} \quad$ Yang, Xinling ${ }^{*, a}$ \\ ( ${ }^{a}$ Department of Applied Chemistry, College of Science, China Agricultural University, Beijing 100193) \\ $\left({ }^{b}\right.$ State Key Laboratory of Integrated Management of Pest Insects and Rodents, Institute of Zoology, \\ Chinese Academy of Sciences, Beijing 100101)
}

\begin{abstract}
As one of the most important sanitation pests worldwide, German cockroach (Blattella germanica L.) is difficult to control. Insect sex pheromones are safe and efficient attractants used in integrated pest management. The sex pheromone of German cockroach had been identified as blattellaquinone. The instability hampered the application of this compound. In order to find new and stable structures, a number of analogues were designed and synthesized by modification of the quinone ring (A), aliphatic terminal (B) and ester group (C) of blattellaquinone. The structures were confirmed by ${ }^{1} \mathrm{H}$ NMR, IR spectra and HRMS analysis.

Keywords Blattella germanica; blattellaquinone; sex pheromone analogues; synthesis
\end{abstract}

德国小蠊是蜚蠊目中分布最广泛、最难治理的卫生 害虫, 不仅是传播多种疾病的重要媒介 ${ }^{[1]}$, 其粪便、表皮 脱落物等还是重要的室内过敏原 ${ }^{[2]}$.

使用高效毒饵是目前防治德国小蠊的主要方法. 高 效毒饵由高效杀虫剂与诱饵混合制成. 长期使用某种杀 虫剂, 使德国小蠊对这些药剂产生了很强的抗性 ${ }^{[3 \sim 5]}$. 高效的诱饵是诱杀蟑螂的前提 ${ }^{[6]}$, 常用的食物诱饵中一 般含有麦芽糖、葡萄糖、果糖和蔗糖，长期使用同一种 诱饵, 德国小蠊会对其中的食物成分产生行为抗性, 表 现为拒食毒饵 ${ }^{[7]}$.

昆虫性信息素是一种天然的引诱物质, 雌性成虫通 过分泌性信息素吸引雄虫前来交配. 2005 年 Nojima 等 ${ }^{[8]}$ 从 15000 头未交配雌成虫尾板的二氯甲烷提取物中, 分 离纯化得到了德国小蠊的性信息素, 确定其结构为含有
一个醌环的异戊酸酯, 将其称为 “小蠊醌” (blattellaquinone, 图 1). 利用性信息素作为引诱剂防治德国小 蠊, 用量少, 引诱效果好, 不仅可以直接诱杀到雄虫, 长期使用还能控制其种群的发展, 不易产生抗性, 而且 不会对非靶标生物有影响，具有较好的应用前景.

德国小蠊的性信息素 1993 年就已经发现 ${ }^{[9,10]}$, 但是 直到 2005 年才确定其结构, 一个重要的原因就是其对 热不稳定; 我们在实验中也发现，常温敞口放置小蠊醌 时, 颜色发生明显变化, 溶解时发现有不溶于常用有机 溶剂的油状物存在. 如果能通过改变 blattellaquinone 的 结构, 使其在保留活性的情况下更稳定, 其应用将更加 安全有效. 基于这一设想, 本文首次对小蠊醌的结构进 行了改造, 分别对 blattellaquinone 的苯醌部分(A)、酯基 部分(B)和末端的脂链部分 $(\mathbf{C})$ 进行了设计, 主要将醌变

*E-mail: yangxl@cau.edu.cn

Received July 28, 2012; revised September 18, 2012; published online September 21, 2012. 


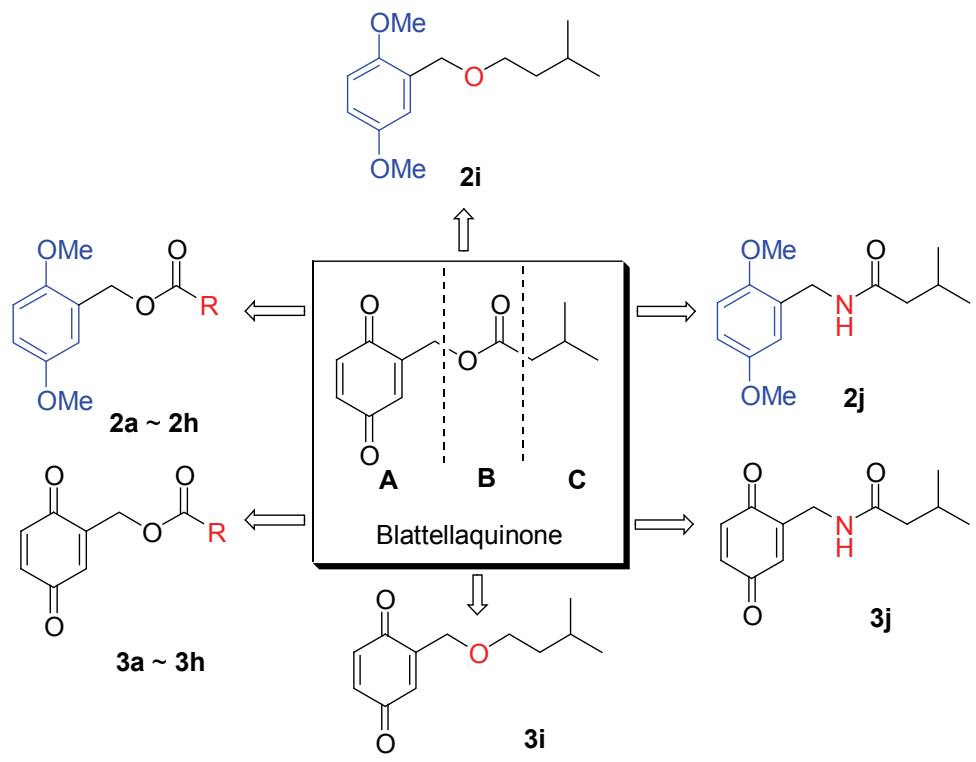

a: $\mathrm{R}=\mathrm{CH}_{3}$, b: $\mathrm{R}=\mathrm{CH}_{2} \mathrm{Cl}$, c: $\mathrm{R}=\mathrm{CH}_{2} \mathrm{CH}_{3}$, d: $\mathrm{R}=\mathrm{CH}_{2} \mathrm{CH}_{2} \mathrm{CH}_{3}$, e: $\mathrm{R}=\left(\mathrm{CH}_{2}\right)_{4} \mathrm{CH}_{3}$,<smiles>[R][R]c1ccc(CC)cc1</smiles>

图 1 目标化合物的设计策略

Figure 1 Design strategy of target compounds

为苯、酯基改换为酰胺及异戊基用其它疏水基替代设计 为进一步的活性评价及构效关系研究奠定基础. 目标物 合成了一系列类似物 $\mathbf{2 a} \sim \mathbf{2} \mathbf{j}$ 和 $\mathbf{3 a} \sim \mathbf{3} \mathbf{j}$ (设计策略见图 1)，的合成路线见 Scheme 1.
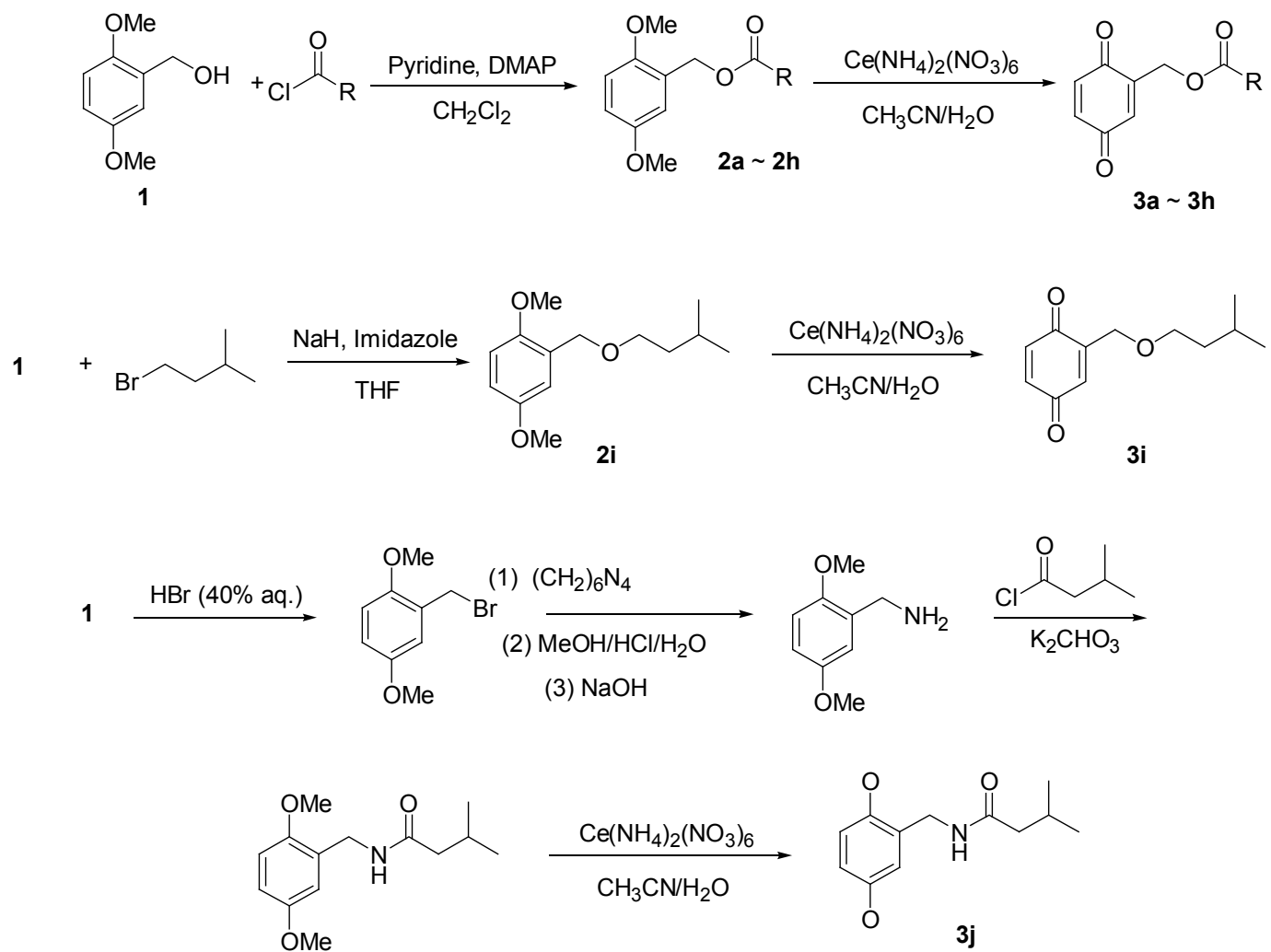

2j

Scheme 1 


\section{1 实验部分}

\section{1 仪器及药品}

实验仪器: Yanagimoto MFG. Co 熔点测定仪, BIBBY/STUART SMP3 熔点测定仪, 温度计未校正; Bruker Avance DPX300 (300 MHz), Bruker DMX600 $(600 \mathrm{MHz})$ 核磁共振仪, 以 TMS 为内标, 氛代氯仿、氛 代二甲基亚砜为溶剂; PerkinElmer Spectrum 100 FT-IR 红外光谱仪; APEX IV 傅立叶变换高分辨质谱. 实验所 用的药品均为市售的分析纯或化学纯，除特别注明外, 未经进一步处理.

\section{2 中间体 $2 \mathrm{a} \sim 2 \mathrm{~h}$ 的合成}

将 2,5-二甲氧基苯甲醇(1) $(2.55 \mathrm{~g}, 15 \mathrm{mmol})$, 吡啶 $(1.98 \mathrm{~g}, 25 \mathrm{mmol}), 4$-二甲氨基吡啶(DMAP) $(2.5 \mathrm{mg}$ )溶 于 $10 \mathrm{~mL}$ 二氯甲烷中, 冰浴冷却至 $0{ }^{\circ} \mathrm{C}$. 保持 $0{ }^{\circ} \mathrm{C}$ 以下 缓慢滴加相应酰氯 $(25 \mathrm{mmol})$, 滴加完后摚拌反应, TLC 检测无原料后停止反应. 加入饱和碳酸氢钠水溶液 除去多余酰氯，乙醚 $(15 \mathrm{~mL} \times 3)$ 萃取后, 1 equiv.盐酸 $(25$ $\mathrm{mL} \times 5$ ) 洗去吡啶, 饱和食盐水洗涤, 无水硫酸钠干燥. 柱层析纯化, 得 $\mathbf{2} \mathbf{a} \sim \mathbf{2} \mathbf{h}^{[8]}$.

乙酸-2,5-二甲氧基苯甲酯(2a): 白色固体，产率 77.8\%. m.p. 41.5 41.7 ${ }^{\circ} \mathrm{C} ;{ }^{1} \mathrm{H}$ NMR $\left(\mathrm{CDCl}_{3}, 300 \mathrm{MHz}\right)$ $\delta: 6.90 \sim 6.92(\mathrm{~m}, 1 \mathrm{H}, \operatorname{ArH}), 6.81(\mathrm{~d}, J=1.59 \mathrm{~Hz}, 2 \mathrm{H}$, $\mathrm{ArH}), 5.13\left(\mathrm{~s}, 2 \mathrm{H}, \mathrm{CH}_{2}\right), 3.78\left(\mathrm{~d}, J=7.14 \mathrm{~Hz}, 6 \mathrm{H}, \mathrm{OCH}_{3}\right)$, 2.10 (s, 3H, $\mathrm{CH}_{3}$ ); IR ( $\left.\mathrm{KBr}\right) v: 3035,2962,2843,1739$, 1613, 1503, 1462, 1255, 1059, 899, 831, $732 \mathrm{~cm}^{-1}$; HRMS calcd for $\mathrm{C}_{11} \mathrm{H}_{14} \mathrm{O}_{4} \mathrm{Na}[\mathrm{M}+\mathrm{Na}]^{+}$: 233.07843 , found 233.07791

2-氯乙酸-2,5-二甲氧基苯甲酯 (2b): 淡黄色液体, 产率 36.2\%. ${ }^{1} \mathrm{H}$ NMR $\left(\mathrm{CDCl}_{3}, 300 \mathrm{MHz}\right) \delta: 6.90 \sim 6.92$ $(\mathrm{m}, 1 \mathrm{H}, \mathrm{ArH}), 6.80 \sim 6.83(\mathrm{~m}, 2 \mathrm{H}, \mathrm{ArH}), 5.24(\mathrm{~s}, 2 \mathrm{H}$, $\left.\mathrm{CH}_{2}\right), 3.78\left(\mathrm{dd}, J=3.18,2.07 \mathrm{~Hz}, 6 \mathrm{H}, \mathrm{OCH}_{3}\right), 4.10(\mathrm{~s}, 2 \mathrm{H}$, $\mathrm{CH}_{2} \mathrm{Cl}$ ); IR (KBr) v: 2956, 2837, 1756, 1593, 1504, 1464, 1222, 1167, 863, 806, $714 \mathrm{~cm}^{-1}$; HRMS calcd for $\mathrm{C}_{11} \mathrm{H}_{13} \mathrm{ClO}_{4} \mathrm{Na}[\mathrm{M}+\mathrm{Na}]^{+}: 267.03946$, found 267.03884 .

丙酸-2,5-二甲氧基苯甲酯(2c): 淡黄色液体, 产率 92.3\%. ${ }^{1} \mathrm{H}$ NMR $\left(\mathrm{CDCl}_{3}, 300 \mathrm{MHz}\right) \delta: 6.91$ (s, $\left.1 \mathrm{H}, \mathrm{ArH}\right)$, $6.80(\mathrm{~d}, J=1.68 \mathrm{~Hz}, 2 \mathrm{H}, \mathrm{ArH}), 5.14\left(\mathrm{~s}, 2 \mathrm{H}, \mathrm{CH}_{2} \mathrm{O}\right), 3.77$ (d, $\left.J=6.99 \mathrm{~Hz}, 6 \mathrm{H}, \mathrm{OCH}_{3}\right), 2.39(\mathrm{q}, J=7.59 \mathrm{~Hz}, 2 \mathrm{H}$, $\left.\mathrm{CH}_{2}\right), 1.16\left(\mathrm{t}, J=7.56 \mathrm{~Hz}, 3 \mathrm{H}, \mathrm{CH}_{3}\right)$; IR (KBr) v: 2944, 2836, 1736, 1593, 1503, 1464, 1221, 1179, 874, 807, 713 $\mathrm{cm}^{-1}$; HRMS calcd for $\mathrm{C}_{12} \mathrm{H}_{16} \mathrm{O}_{4} \mathrm{Na}[\mathrm{M}+\mathrm{Na}]^{+}: 247.09408$, found 247.09345 .

丁酸-2,5-二甲氧基苯甲酯(2d): 淡黄色液体, 产率 94.3\%. ${ }^{1} \mathrm{H} \mathrm{NMR}\left(\mathrm{CDCl}_{3}, 300 \mathrm{MHz}\right) \delta: 6.90$ (t, $J=1.80 \mathrm{~Hz}$,
$1 \mathrm{H}, \operatorname{ArH}), 6.80(\mathrm{~d}, J=1.71 \mathrm{~Hz}, 2 \mathrm{H}, \operatorname{ArH}), 5.14(\mathrm{~s}, 2 \mathrm{H}$, $\left.\mathrm{CH}_{2} \mathrm{O}\right), 3.77\left(\mathrm{~d}, J=6.57 \mathrm{~Hz}, 6 \mathrm{H}, \mathrm{OCH}_{3}\right), 2.32 \sim 2.35(\mathrm{~m}$, $2 \mathrm{H}, \mathrm{CH}_{2} \mathrm{CH}_{2}$ ), $1.62 \sim 1.75\left(\mathrm{~m}, 2 \mathrm{H}, \mathrm{CH}_{2} \mathrm{CH}_{3}\right), 0.95$ (t, $J=$ $7.35 \mathrm{~Hz}, 3 \mathrm{H}, \mathrm{CH}_{3}$ ); IR (KBr) v: 2964, 2836, 1735, 1593, 1503, 1464, 1221, 1176, 873, 806, $713 \mathrm{~cm}^{-1}$; HRMS calcd for $\mathrm{C}_{13} \mathrm{H}_{18} \mathrm{O}_{4} \mathrm{Na}[\mathrm{M}+\mathrm{Na}]^{+}:$261.10973, found 261.10925.

己酸-2,5-二甲氧基苯甲酯 (2e): 淡黄色液体，产 61.8\%. ${ }^{1} \mathrm{H}$ NMR $\left(\mathrm{CDCl}_{3}, 600 \mathrm{MHz}\right) \delta: 7.03(\mathrm{~s}, 1 \mathrm{H}, \mathrm{ArH})$, $6.93(\mathrm{~d}, J=1.29 \mathrm{~Hz}, 2 \mathrm{H}, \mathrm{ArH}), 5.26\left(\mathrm{~s}, 2 \mathrm{H}, \mathrm{CH}_{2} \mathrm{O}\right), 3.90$ (d, $\left.J=13.75 \mathrm{~Hz}, 6 \mathrm{H}, \mathrm{OCH}_{3}\right), 2.48(\mathrm{t}, J=7.46 \mathrm{~Hz}, 2 \mathrm{H}$, $\left.\mathrm{COCH}_{2}\right), 1.76 \sim 1.81\left(\mathrm{~m}, 2 \mathrm{H}, \mathrm{CH}_{2}\right), 1.38 \sim 1.46(\mathrm{~m}, 4 \mathrm{H}$, $\left.\mathrm{CH}_{2} \mathrm{CH}_{2}\right), 1.01\left(\mathrm{t}, J=6.70 \mathrm{~Hz}, 3 \mathrm{H}, \mathrm{CH}_{3}\right)$; IR $(\mathrm{KBr}) v$ : 2955, 2871, 2836, 1736, 1593, 1502, 1465, 1221, 1167, $874,805,713 \mathrm{~cm}^{-1}$; HRMS calcd for $\mathrm{C}_{15} \mathrm{H}_{22} \mathrm{O}_{4} \mathrm{Na}[\mathrm{M}+$ $\mathrm{Na}]^{+}: 289.13941$, found 289.14045 .

苯甲酸-2,5-二甲氧基苯甲酯(2f): 白色固体，产率 $96.1 \%$. m.p. $66 \sim 67{ }^{\circ} \mathrm{C}$ (文献值 ${ }^{[11]}$ m.p. $62 \sim 63{ }^{\circ} \mathrm{C}$ ); ${ }^{1} \mathrm{H}$ NMR $\left(\mathrm{CDCl}_{3}, 600 \mathrm{MHz}\right) \delta: 8.21(\mathrm{~d}, J=7.53 \mathrm{~Hz}, 2 \mathrm{H}$, $\mathrm{ArH}), 7.68$ (t, $J=7.36 \mathrm{~Hz}, 1 \mathrm{H}, \mathrm{ArH}), 7.56(\mathrm{t}, J=7.73 \mathrm{~Hz}$, $2 \mathrm{H}, \mathrm{ArH}), 7.14(\mathrm{~s}, 1 \mathrm{H}, \mathrm{ArH}), 7.69(\mathrm{~s}, 2 \mathrm{H}, \mathrm{ArH}), 5.51(\mathrm{~s}$, $\left.2 \mathrm{H}, \mathrm{CH}_{2} \mathrm{O}\right), 3.92\left(\mathrm{~d}, J=24.3 \mathrm{~Hz}, 6 \mathrm{H}, \mathrm{OCH}_{3}\right)$; IR $(\mathrm{KBr}) v$ : 2998, 2939, 2833, 1718, 1597, 1498, 1459, 1277, 1220, $863,795,717 \mathrm{~cm}^{-1}$; HRMS calcd for $\mathrm{C}_{16} \mathrm{H}_{16} \mathrm{O}_{4} \mathrm{Na}[\mathrm{M}+$ $\mathrm{Na}]^{+}: 295.09408$, found 295.09399 .

苯乙酸-2,5-二甲氧基苯甲酯 $(\mathbf{2 g})$ : 淡黄色液体，产 率 62.9\%; ${ }^{1} \mathrm{H}$ NMR (DMSO- $\left.d_{6}, 600 \mathrm{MHz}\right) \delta: 7.36 \sim 7.42$ $(\mathrm{m}, 5 \mathrm{H}, \mathrm{ArH}), 6.91 \sim 7.04(\mathrm{~m}, 3 \mathrm{H}, \operatorname{ArH}), 5.15(\mathrm{~s}, 2 \mathrm{H}$, $\left.\mathrm{CH}_{2} \mathrm{O}\right), 3.82\left(\mathrm{~d}, J=6.01 \mathrm{~Hz}, 6 \mathrm{H}, \mathrm{OCH}_{3}\right), 3.75(\mathrm{~s}, 2 \mathrm{H}$, $\mathrm{CH}_{2}$ ); IR (KBr) v: 2940, 2909, 2834, 1741, 1600, 1502, $1462,1277,1219,964,812,724 \mathrm{~cm}^{-1}$; HRMS calcd for $\mathrm{C}_{34} \mathrm{H}_{36} \mathrm{O}_{8} \mathrm{Na}[2 \mathrm{M}+\mathrm{Na}]^{+}:$595.23024, found 595.23032.

环丙甲酸-2,5-二甲氧基苯甲酯(2h): 白色固体，产 率 87.6\%. m.p. $54 \sim 55{ }^{\circ} \mathrm{C} ;{ }^{1} \mathrm{H}$ NMR $\left(\mathrm{CDCl}_{3}, 300 \mathrm{MHz}\right) \delta$ : $6.92(\mathrm{t}, J=1.72 \mathrm{~Hz}, 1 \mathrm{H}, \mathrm{ArH}), 6.82(\mathrm{~d}, J=1.73 \mathrm{~Hz}, 2 \mathrm{H}$, $\mathrm{ArH}), 5.15\left(\mathrm{~d}, J=0.47 \mathrm{~Hz}, 2 \mathrm{H}, \mathrm{CH}_{2} \mathrm{O}\right), 3.80(\mathrm{~d}, J=6.71$ $\left.\mathrm{Hz}, 6 \mathrm{H}, \mathrm{OCH}_{3}\right), 1.64 \sim 1.72(\mathrm{~m}, 1 \mathrm{H}, \mathrm{CH}), 1.01 \sim 1.07(\mathrm{~m}$, $\left.2 \mathrm{H}, \mathrm{CH}_{2}\right), 0.84 \sim 0.90\left(\mathrm{~m}, 2 \mathrm{H}, \mathrm{CH}_{2}\right)$; IR (KBr) v: 3414 , 2953, 2835, 1719, 1591, 1505, 1455, 1223, 1178, 886, 808, $712 \mathrm{~cm}^{-1}$; HRMS calcd for $\mathrm{C}_{13} \mathrm{H}_{16} \mathrm{O}_{4} \mathrm{Na}[\mathrm{M}+\mathrm{Na}]^{+}$: 259.09408 , found 259.09355 .

\section{3 目标产物 $3 a \sim 3 h$ 的合成}

硝酸铈铵(13.16 g, $24 \mathrm{mmol}$ )溶于 $50 \mathrm{~mL}$ 水中; 将 $\mathbf{2 a} \sim 2 \mathbf{h}(8 \mathrm{mmol})$ 溶于 $40 \mathrm{~mL}$ 乙腈后，室温搅拌下滴入上 述水溶液中, $1 \sim 3 \mathrm{~h}$ 后检测反应完成. 混合物用乙醚 $(50$ $\mathrm{mL} \times 3)$ 萃取, 有机层加入 $150 \mathrm{~mL}$ 碳酸氢钠水溶液摚拌 
$20 \mathrm{~min}$, 分液后有机层用饱和食盐水洗涤, 无水硫酸钠 干燥. 脱溶, 柱层析纯化, 得 $\mathbf{3 a} \sim \mathbf{3} \mathbf{h}^{[8]}$.

乙酸-(3,6-二酮-1,4-环己二烯)-甲基酯(3a): 黄色固 体, 产率 $41.0 \%$. m.p. $120.7 \sim 121.0{ }^{\circ} \mathrm{C} ;{ }^{1} \mathrm{H}$ NMR $\left(\right.$ DMSO- $\left.d_{6}, 300 \mathrm{MHz}\right) \delta: 6.85 \sim 6.94(\mathrm{~m}, 2 \mathrm{H}, \mathrm{CH}=\mathrm{CH})$, $6.70 \sim 6.72(\mathrm{~m}, 1 \mathrm{H}, \mathrm{CH}), 4.89 \sim 4.90\left(\mathrm{~m}, 2 \mathrm{H}, \mathrm{CH}_{2} \mathrm{O}\right), 2.12$ (s, 3H, $\left.\mathrm{CH}_{3}\right)$; IR (KBr) v: 2359, 1715, 1652, 1268, 713 $\mathrm{cm}^{-1}$; HRMS calcd for $\mathrm{C}_{11} \mathrm{H}_{14} \mathrm{O}_{4} \mathrm{Na}[\mathrm{M}+\mathrm{Na}]^{+}$: 203.03107, found 203.03107.

2-氯乙酸-(3,6-二酮-1,4-环己二烯)-甲基酯(3b): 黄 色固体, 产率 36.6\%. m.p. $103 \sim 104{ }^{\circ} \mathrm{C} ;{ }^{1} \mathrm{H}$ NMR $\left(\mathrm{DMSO}-d_{6}, 600 \mathrm{MHz}\right) \delta: 6.88 \sim 6.94(\mathrm{~m}, 2 \mathrm{H}, \mathrm{CH}=\mathrm{CH})$, $6.79(\mathrm{~s}, 1 \mathrm{H}, \mathrm{CH}), 5.03\left(\mathrm{~s}, 2 \mathrm{H}, \mathrm{CH}_{2} \mathrm{O}\right), 4.57\left(\mathrm{~s}, 2 \mathrm{H}, \mathrm{CH}_{2} \mathrm{Cl}\right)$; IR (KBr) v: 3714, 3057, 2934, 2851, 1760, 1662, 1601, 1207, 1184, $929 \mathrm{~cm}^{-1}$; HRMS calcd for $\mathrm{C}_{9} \mathrm{H}_{7} \mathrm{ClO}_{4} \mathrm{Na}$ $[\mathrm{M}+\mathrm{Na}]^{+}:$236.99251, found 236.99232 .

丙酸-(3,6-二酮-1,4-环己二烯)-甲基酯(3c): 黄色固 体, 产率 43.9\%. m.p. 81 82 ${ }^{\circ} \mathrm{C} ;{ }^{1} \mathrm{H}$ NMR (DMSO- $d_{6}$, $600 \mathrm{MHz}) \delta: 6.96 \sim 7.03(\mathrm{~m}, 2 \mathrm{H}, \mathrm{CH}=\mathrm{CH}), 6.78 \sim 6.79$ $(\mathrm{m}, 1 \mathrm{H}, \mathrm{CH}), 5.01\left(\mathrm{~d}, J=1.37 \mathrm{~Hz}, 2 \mathrm{H}, \mathrm{CH}_{2} \mathrm{O}\right), 2.55$ (q, $J=$ $7.45 \mathrm{~Hz}, 2 \mathrm{H}, \mathrm{CH}_{2} \mathrm{CH}_{3}$ ), 1.16 (t, $J=7.45 \mathrm{~Hz}, 3 \mathrm{H}, \mathrm{CH}_{3}$ ); IR (KBr) $v$ : 3684, 3073, 2983, 2944, 1753, 1656, 1603, 1194, $947 \mathrm{~cm}^{-1}$; HRMS calcd for $\mathrm{C}_{10} \mathrm{H}_{10} \mathrm{O}_{4} \mathrm{Na}[\mathrm{M}+\mathrm{Na}]^{+}$: 217.04713, found 217.04669.

丁酸-(3,6-二酮-1,4-环己二烯)-甲基酯(3d): 黄色固 体, 产率 96.1\%. m.p. $66 \sim 67{ }^{\circ} \mathrm{C} ;{ }^{1} \mathrm{H}$ NMR $\left(\mathrm{CDCl}_{3}, 600\right.$ MHz) $\delta: 6.88 \sim 6.92(\mathrm{~m}, 2 \mathrm{H}, \mathrm{CH}=\mathrm{CH}), 6.80(\mathrm{~d}, J=1.90$ $\mathrm{Hz}, 1 \mathrm{H}, \mathrm{CH}), 5.12\left(\mathrm{~d}, J=1.74 \mathrm{~Hz}, 2 \mathrm{H}, \mathrm{CH}_{2} \mathrm{O}\right), 2.52(\mathrm{t}, J=$ $\left.7.49 \mathrm{~Hz}, 2 \mathrm{H}, \mathrm{COCH}_{2}\right), 1.80 \sim 1.85\left(\mathrm{~m}, 2 \mathrm{H}, \mathrm{CH}_{2} \mathrm{CH}_{3}\right), 1.10$ (t, $J=7.39 \mathrm{~Hz}, 3 \mathrm{H}, \mathrm{CH}_{3}$ ); IR (KBr) v: 3057, 2970, 2361, 1749, 1645, 1173, 928, $427 \mathrm{~cm}^{-1}$; HRMS calcd for $\mathrm{C}_{13} \mathrm{H}_{18} \mathrm{O}_{4} \mathrm{Na}[\mathrm{M}+\mathrm{Na}]^{+}: 231.06278$, found 231.06247.

己酸-(3,6-二酮-1,4-环己二烯)-甲基酯(3e): 黄色固 体, 产率 32.5\%. m.p. $40 \sim 41{ }^{\circ} \mathrm{C} ;{ }^{1} \mathrm{H}$ NMR (DMSO- $d_{6}$, $600 \mathrm{MHz}) \delta: 6.97 \sim 7.03(\mathrm{~m}, 2 \mathrm{H}, \mathrm{CH}=\mathrm{CH}), 6.78(\mathrm{~s}, 1 \mathrm{H}$, $\mathrm{CH}), 5.01\left(\mathrm{~s}, 2 \mathrm{H}, \mathrm{CH}_{2} \mathrm{O}\right), 2.52(\mathrm{t}, J=7.42 \mathrm{~Hz}, 2 \mathrm{H}$, $\left.\mathrm{COCH}_{2}\right), 1.64 \sim 1.67\left(\mathrm{~m}, 2 \mathrm{H}, \mathrm{CH}_{2}\right), 1.34 \sim 1.38(\mathrm{~m}, 4 \mathrm{H}$, $\left.\mathrm{CH}_{2} \mathrm{CH}_{2}\right), 0.96\left(\mathrm{t}, J=6.60 \mathrm{~Hz}, 3 \mathrm{H}, \mathrm{CH}_{3}\right)$; IR ( $\left.\mathrm{KBr}\right) v$ : 3700, 3069, 2969, 2934, 2871, 1750, 1657, 1613, 1327, $1171,936 \mathrm{~cm}^{-1}$; HRMS calcd for $\mathrm{C}_{13} \mathrm{H}_{16} \mathrm{O}_{4} \mathrm{Na}[\mathrm{M}+\mathrm{Na}]^{+}$: 259.09246, found 259.09367 .

苯甲酸-(3,6-二酮-1,4-环己二烯)-甲基酯(3f): 黄色 固体, 产率 30.0\%. m.p. 66 67 ${ }^{\circ} \mathrm{C} ;{ }^{1} \mathrm{H}$ NMR (DMSO- $d_{6}$, $600 \mathrm{MHz}) \delta: 8.15(\mathrm{~d}, J=7.73 \mathrm{~Hz}, 2 \mathrm{H}, \mathrm{ArH}), 7.79 \sim 7.82$ (m, 1H, ArH), $7.66 \sim 7.68(\mathrm{~m}, 2 \mathrm{H}, \operatorname{ArH}), 7.00 \sim 7.07(\mathrm{~m}$,
$2 \mathrm{H}, \mathrm{CH}=\mathrm{CH}), 6.93(\mathrm{~s}, 1 \mathrm{H}, \mathrm{CH}), 5.28\left(\mathrm{~s}, 2 \mathrm{H}, \mathrm{CH}_{2} \mathrm{O}\right) ; \mathrm{IR}$ (KBr) v: 3057, 1743, 1645, 1245, 1044, 934, $427 \mathrm{~cm}^{-1}$; HRMS calcd for $\mathrm{C}_{14} \mathrm{H}_{10} \mathrm{O}_{4} \mathrm{Na}[\mathrm{M}+\mathrm{Na}]^{+}:$265.04713, found 265.04700 .

苯乙酸-(3,6-二酮-1,4-环己二烯)-甲基酯(3g)：黄色 固体, 产率 69.3\%. m.p. $55 \sim 56{ }^{\circ} \mathrm{C} ;{ }^{1} \mathrm{H}$ NMR (DMSO- $d_{6}$, $600 \mathrm{MHz}) \delta: 7.38 \sim 7.45(\mathrm{~m}, 5 \mathrm{H}, \mathrm{ArH}), 6.93 \sim 7.02(\mathrm{~m}$, $2 \mathrm{H}, \mathrm{CH}=\mathrm{CH}), 6.70 \sim 6.74(\mathrm{~m}, 1 \mathrm{H}, \mathrm{CH}), 5.04(\mathrm{~s}, 2 \mathrm{H}$, $\mathrm{CH}_{2} \mathrm{O}$ ), 3.92 (s, 2H, $\mathrm{CH}_{2}$ ); IR (KBr) v: 3738, 3059, 2929, 2871, 1736, 1655, 1614, 1518, 1465, 1430, 1181, 951, 776, $718 \mathrm{~cm}^{-1}$; HRMS calcd for $\mathrm{C}_{15} \mathrm{H}_{12} \mathrm{O}_{4} \mathrm{Na}[\mathrm{M}+\mathrm{Na}]^{+}$: 279.06278 , found 279.06249 .

环丙甲酸-(3,6-二酮-1,4-环己二烯)-甲基酯(3h): 黄 色固体, 产率 93.9\%. m.p. $106 \sim 107{ }^{\circ} \mathrm{C} ;{ }^{1} \mathrm{H}$ NMR $\left(\right.$ DMSO- $\left.d_{6}, 600 \mathrm{MHz}\right) \delta: 6.97 \sim 7.03(\mathrm{~m}, 2 \mathrm{H}, \mathrm{CH}=\mathrm{CH})$, $6.78(\mathrm{~d}, J=1.80 \mathrm{~Hz}, 1 \mathrm{H}, \mathrm{CH}), 5.01\left(\mathrm{~s}, 2 \mathrm{H}, \mathrm{CH}_{2} \mathrm{O}\right), 1.85 \sim$ $1.89(\mathrm{~m}, 1 \mathrm{H}, \mathrm{CH}), 1.04 \sim 1.07\left(\mathrm{~m}, 2 \mathrm{H}, \mathrm{CH}_{2}\right), 0.98 \sim 1.00$ (m, 2H, $\mathrm{CH}_{2}$ ); IR (KBr) v: 3738, 3062, 3053, 2920, 1732, 1656, 1603, 1174, $941 \mathrm{~cm}^{-1}$; HRMS calcd for $\mathrm{C}_{11} \mathrm{H}_{10} \mathrm{O}_{4} \mathrm{Na}$ $[\mathrm{M}+\mathrm{Na}]^{+}: 229.04713$, found 229.04674 .

1.42 -异戊氧基-甲基-1,4-二甲氧基苯(2i)和 2-异戊氧 基-甲基-3,6-二酮-1,4-环己二烯(3i)的合成

\subsubsection{2-异戊氧基-甲基-1, 4-二甲氧基苯(2i)的合成}

2,5-二甲氧基苯甲醇 $(1.50 \mathrm{~g}, 8.93 \mathrm{mmol}$ ), 咪唑(20 $\mathrm{mg})$, 氢化钠 $(60 \%, 1.1 \mathrm{~g}, 26.79 \mathrm{mmol}$,)溶于 $15 \mathrm{~mL}$ 无水 四氢呋喃, 氮气保护, 室温反应 $3 \mathrm{~h}$. 滴加 1-溴-3-甲基丁烷 $(5.4 \mathrm{~g}, 35.72 \mathrm{mmol})$, 反应 $6 \mathrm{~h}$ 后加入 $20 \mathrm{~mL}$ 去离子 水淬灭反应，二氯甲烷 $(30 \mathrm{~mL} \times 3)$ 萃取后，饱和食盐水 洗涤, 无水硫酸钠干燥. 柱层析纯化 $[V$ (乙醚) : $V$ (石油 醚 $)=1: 2]$, 得 $1.18 \mathrm{~g}$ 淡黄色液体 $\mathbf{2 i}^{[11,12]}$, 收率 $55.4 \%$. ${ }^{1} \mathrm{H}$ NMR $\left(\mathrm{CDCl}_{3}, 600 \mathrm{MHz}\right) \delta: 7.12(\mathrm{~d}, J=2.39 \mathrm{~Hz}, 1 \mathrm{H}$, $\mathrm{ArH}), 6.86 \sim 6.91(\mathrm{~m}, 2 \mathrm{H}, \mathrm{ArH}), 4.64\left(\mathrm{~s}, 2 \mathrm{H}, \mathrm{CH}_{2} \mathrm{O}\right), 3.90$ (d, $\left.J=2.90 \mathrm{~Hz}, 6 \mathrm{H}, \mathrm{OCH}_{3}\right), 3.66(\mathrm{t}, J=6.69 \mathrm{~Hz}, 2 \mathrm{H}$, $\mathrm{OCH}_{2}$ ), $1.85 \sim 1.88\left(\mathrm{~m}, 1 \mathrm{H}, \mathrm{CH}\right.$ ), 1.37 (brs, $2 \mathrm{H}, \mathrm{CH}_{2}$ ), $1.02 \sim 1.03$ (d, $J=6.62 \mathrm{~Hz}, 6 \mathrm{H}, \mathrm{CH}_{3}$ ); IR (KBr) v: 2960, 2883, 2849, 1603, 1501, 1479, 1273, 874, 807, $72 \mathrm{~cm}^{-1}$; HRMS calcd for $\mathrm{C}_{14} \mathrm{H}_{22} \mathrm{O}_{3} \mathrm{Na}[\mathrm{M}+\mathrm{Na}]^{+}:$261.14612, found 261.14623 .

\subsubsection{2-异戊氧基-甲基-3,6-二酮-1,4-环己二烯(3i)的} 合成

硝酸铈铵(13.16 g, $24 \mathrm{mmol}$ )溶于 $50 \mathrm{~mL}$ 水中; 将 $\mathbf{2 i}$ $(2.3 \mathrm{~g}, 8 \mathrm{mmol})$ 溶于 $40 \mathrm{~mL}$ 乙腈后, 室温搅拌下滴入上 述水溶液中, $30 \mathrm{~min}$ 后检测反应完成. 混合物用乙醚(50 $\mathrm{mL} \times 3$ ) 萃取, 有机层加入 $150 \mathrm{~mL}$ 碳酸氢钠水溶液搅拌 $20 \mathrm{~min}$, 分液后有机层用饱和食盐水洗涤, 无水硫酸钠 
干燥, 柱层析纯化 $[V$ (乙醚) $: V$ (石油醚) $=1: 2]$, 得黄色 油状液滴 $0.14 \mathrm{~g}^{[11,12]}$, 收率 8.4\%. ${ }^{1} \mathrm{H}$ NMR (DMSO- $d_{6}$, $600 \mathrm{MHz}) \delta: 6.93 \sim 6.98(\mathrm{~m}, 2 \mathrm{H}, \mathrm{CH}=\mathrm{CH}), 6.71(\mathrm{~s}, 1 \mathrm{H}$, $\mathrm{CH}), 4.38\left(\mathrm{~s}, 2 \mathrm{H}, \mathrm{CH}_{2} \mathrm{O}\right), 3.63$ (t, $J=6.61 \mathrm{~Hz}, 2 \mathrm{H}, \mathrm{OCH}_{2}$ ), $1.76 \sim 1.80(\mathrm{~m}, 1 \mathrm{H}, \mathrm{CH}), 1.54 \sim 1.57\left(\mathrm{~m}, 2 \mathrm{H}, \mathrm{CH}_{2}\right), 0.98$ (d, $J=6.59 \mathrm{~Hz}, 6 \mathrm{H}, \mathrm{CH}_{3}$ ); IR ( $\left.\mathrm{KBr}\right) v$ : 3529, 3423, 2988, 2920, 2875, 2847, 1714, 1660, 1602, 1367, 1229, 912 $\mathrm{cm}^{-1}$; HRMS calcd for $\mathrm{C}_{24} \mathrm{H}_{32} \mathrm{O}_{6} \mathrm{Na}[2 \mathrm{M}+\mathrm{Na}]^{+}$: 439.20876, found 439.20911.

\section{$1.5 \mathrm{~N}-$-(2,5-二甲氧基苯甲基)-异戊酰胺(2j)和 $N-[(3,6-$} 二酮-1,4-环己二烯)-甲基]-异戊酰胺(3j)的合成

\subsubsection{2,5-二甲氧基芐溴的合成}

氢溴酸 $(40 \%, 20 \mathrm{~mL}) 0{ }^{\circ} \mathrm{C}$ 下加入到 2,5-二甲氧基苯 甲醇 $(7 \mathrm{~g}, 42 \mathrm{mmol})$ 中, 保持 $0{ }^{\circ} \mathrm{C}$ 反应 $0.5 \mathrm{~h}$, 二氯甲烷 $(50 \mathrm{~mL} \times 3)$ 萃取, 有机层分别用饱和碳酸氢钠, 水, 饱 和食盐水洗涤, 无水硫酸钠干燥, 柱层析纯化 $[V($ 乙 醚) $: V$ (石油醚 $)=1: 3.5]$, 得 $2.97 \mathrm{~g}$ 白色固 体 $^{[13,14]}$, 收 率 $30.6 \%$.

\subsubsection{2,5-二甲氧基芐胺的合成}

将六次甲基四胺(乌洛托品)(2.1 g, $15.0 \mathrm{mmol}$ )溶于 $60 \mathrm{~mL}$ 氯仿中, 加热至回流; 2,5-二甲氧基芐溴 $(2.97 \mathrm{~g}$, $12.9 \mathrm{mmol}$ )溶于 $60 \mathrm{~mL}$ 氯仿后, 于 $30 \mathrm{~min}$ 内滴入乌洛托 品中, 回流 $3 \mathrm{~h}, 4{ }^{\circ} \mathrm{C}$ 储存过夜, 有白色固体析出. 过滤 后得 $2.33 \mathrm{~g}$ 白色固体. 将上述固体溶于混合液中 $[V($ 甲 醇) $: V($ 浓盐酸 $): V($ 水 $)=42: 12: 10]$, 加热回流 $3 \mathrm{~d}$. 浓缩后加入 $10 \mathrm{~mL}$ 水溶解, 加入 $6 \mathrm{~mol} / \mathrm{L}$ 氢氧化钠水溶 液(约 $40 \mathrm{~mL}$ ) 调节 $\mathrm{pH}$ 至中性, 乙醚 $(50 \mathrm{~mL} \times 3$ )萃取后, 饱和食盐水洗涤, 无水碳酸钾干燥. 浓缩后得 $0.96 \mathrm{~g}$ 粗 产物胺, 收率 $44.5 \%$.

\subsection{3 $\mathrm{N}$-(2,5-二甲氧基苯甲基)-异戊酰胺 $(\mathbf{2} \mathbf{j})$ 的合成}

2,5-二甲氧基芐胺 $(2.0 \mathrm{~g}, 12 \mathrm{mmol}$ )溶于 $15 \mathrm{~mL}$ 二氯 甲烷中, 加入 $2.1 \mathrm{~g}$ 无水碳酸钾, 保持 $0 \sim-5{ }^{\circ} \mathrm{C}$, 滴加 异戊酰氯( $1.8 \mathrm{~g}, 15 \mathrm{mmol})$, 常温摚拌反应 $2 \mathrm{~d}$. 乙醚(30 $\mathrm{mL} \times 3$ )萃取后饱和食盐水洗涤, 无水碳酸钾干燥后脱 溶, 得 $1.32 \mathrm{~g}$ 白色固体, 收率 43.7\%, m.p. 79 $80{ }^{\circ} \mathrm{C} ;{ }^{1} \mathrm{H}$ NMR $\left(\mathrm{CDCl}_{3}, 600 \mathrm{MHz}\right) \delta: 6.98(\mathrm{~d}, J=1.47 \mathrm{~Hz}, 1 \mathrm{H}$, ArH), $6.91 \sim 6.93(\mathrm{~m}, 2 \mathrm{H}, \mathrm{ArH}), 5.99$ (brs, 1H, NH), 4.54 (d, $\left.J=5.42 \mathrm{~Hz}, 2 \mathrm{H}, \mathrm{CH}_{2} \mathrm{NH}\right), 3.93\left(\mathrm{~s}, 3 \mathrm{H}, \mathrm{OCH}_{3}\right), 3.88$ (s, $\left.3 \mathrm{H}, \mathrm{OCH}_{3}\right), 2.23 \sim 2.25(\mathrm{~m}, 1 \mathrm{H}, \mathrm{CH}), 2.18(\mathrm{~d}, J=7.08 \mathrm{~Hz}$, $\left.2 \mathrm{H}, \mathrm{CH}_{2}\right), 1.06$ [d, $\left.J=6.49 \mathrm{~Hz}, 6 \mathrm{H}, \mathrm{CH}\left(\mathrm{CH}_{3}\right)_{2}\right]$; IR $(\mathrm{KBr})$ $v: 3450,2933,2879,1613,1570,1498,1460,1086,1049$, $876,825,713 \mathrm{~cm}^{-1}$; HRMS calcd for $\mathrm{C}_{14} \mathrm{H}_{21} \mathrm{NO}_{3} \mathrm{Na}[\mathrm{M}+$ $\mathrm{Na}]^{+}: 274.14136$, found 274.14148 .
1.5.4 $N$-[(3,6-二酮-1,4-环已二烯 $)$-甲基]-异戊酰胺 (3j)的合成

硝酸铈铵(13.16 g, $24 \mathrm{mmol}$ )溶于 $50 \mathrm{~mL}$ 水中; 将 $\mathbf{2 j}$ (2.01 g, $8 \mathrm{mmol}$ )溶于 $40 \mathrm{~mL}$ 乙腈后, 室温摚拌下滴入上 述水溶液中, $3 \mathrm{~h}$ 后检测反应完成. 混合物用乙醚 $(50$ $\mathrm{mL} \times 3$ ) 萃取, 有机层加入 $150 \mathrm{~mL}$ 碳酸氢钠水溶液搅拌 20 分钟, 分液后有机层用饱和食盐水洗涤, 无水硫酸钠 干燥, 柱层析纯化 $[V$ (甲醇) $: V$ (二氯甲烷) $=1: 99$ ], 得 黄色固体 1.45 g, 收率 $81.9 \%$, m.p. $103 \sim 104{ }^{\circ} \mathrm{C} ;{ }^{1} \mathrm{H}$ NMR (DMSO- $\left.d_{6}, 600 \mathrm{MHz}\right) \delta: 6.57(\mathrm{~d}, J=1.97 \mathrm{~Hz}, 1 \mathrm{H}$, $\mathrm{CH}), 6.93 \sim 7.01(\mathrm{~m}, 2 \mathrm{H}, \mathrm{CH}=\mathrm{CH}), 5.86(\mathrm{br}, 1 \mathrm{H}, \mathrm{NH})$, $4.14\left(\mathrm{~d}, J=1.21 \mathrm{~Hz}, 2 \mathrm{H}, \mathrm{CH}_{2} \mathrm{NH}\right), 2.03 \sim 2.09(\mathrm{~m}, 1 \mathrm{H}$, $\mathrm{CH}), 2.11 \sim 2.18\left(\mathrm{~m}, 2 \mathrm{H}, \mathrm{CH}_{2}\right), 1.00[\mathrm{~d}, J=6.65 \mathrm{~Hz}, 6 \mathrm{H}$, $\left.\mathrm{CH}\left(\mathrm{CH}_{3}\right)_{2}\right]$; IR (KBr) v: 3289, 3099, 2976, 2885, 1656, 1601, 1562, $948 \mathrm{~cm}^{-1}$; HRMS calcd for $\mathrm{C}_{12} \mathrm{H}_{15} \mathrm{NO}_{3} \mathrm{Na}$ $[\mathrm{M}+\mathrm{Na}]^{+}: 244.09411$, found 244.09403 .

\section{2 结果与讨论}

\section{1 目标化合物的合成}

以化合物 1 (2,5-二甲氧基苯甲醇)为起始原料，经 与相应的酰氯一步反应，即可制得 $\mathbf{2 a} \sim \mathbf{2 h}$. 合成中所用 的缚酸剂吡啶也可用无水碳酸钾代替，使用吡啶反应时 间快(1 6 h), 放热剧烈, 副反应多, 需要用冰浴将温度 控制在 $0{ }^{\circ} \mathrm{C}$ 以下; 用无水碳酸钾做缚酸剂则反应温和, 室温即可，但是反应时间将会显著增加( $1 \mathrm{~d})$. 用吡啶做 缚酸剂时, 用文献 ${ }^{[8]}$ 的后处理方法并不能将其洗去, 用 $1 \mathrm{~mol} / \mathrm{L}$ 盐酸洗涤数次可基本除去有机层中的吡啶，可 作为粗产物直接进行下一步反应. $\mathbf{2 a} \sim 2 \mathrm{~h}$ 在硝酸铈铵作 用下进一步氧化，即可得到 $\mathbf{3 a} \sim \mathbf{3 h}$.

由 $2 \mathbf{i}$ 制备 3i 时收率很低，只有 $8.4 \%$, 原因可能是 因为氧化过程中发生了如 Scheme 2 所示的反应 ${ }^{[12]}$, 产 物 4 的收率在 46\% 97\%(取代基的不同)，而相应的产 物醌 5 只有 $1 \% \sim 5 \%$.
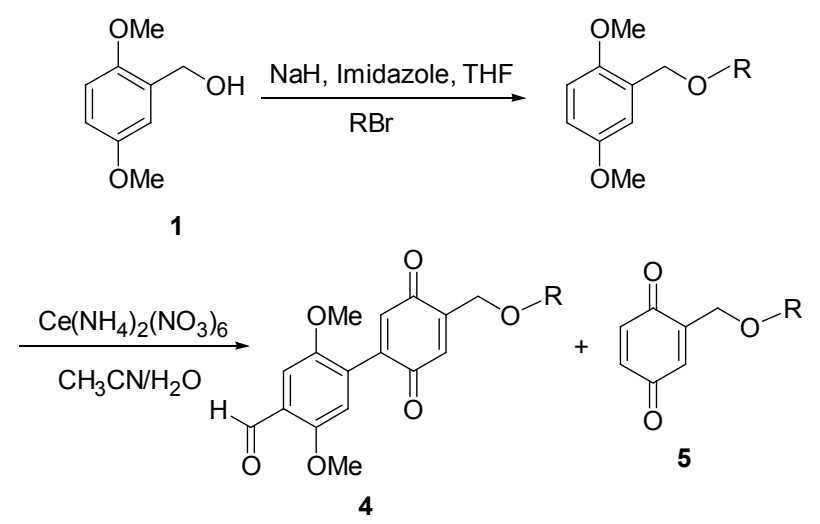

R = Butyl, Hexyl, Decyl, Tridecyl, Texadecyl, Hexadecyl, Octadecyl 
$2 \mathbf{j}$ 和 $3 \mathbf{j}$ 的制备则是由 2,5 -二甲氧基苯甲醇为起始原 料, 经氢溴酸溴化, 六次甲基四胺氨化, 再与异成酰氯 反应即可得到 $2 \mathbf{j}, 2 \mathbf{j}$ 在硝酸铈铵作用下进一步氧化, 即 可以较高收率得到 $\mathbf{3 j}$.

\section{2 目标化合物的波谱分析}

以目标物 $3 \mathbf{h}$ 为例, 对其谱图进行解析. 在 $\mathbf{~ h ~}$ 的 ${ }^{1} \mathrm{H}$ NMR 谱图中, 醌环上 C-1, C-2 上的 $\mathrm{H}$, 化学位移在 $\delta$ 6.97 7.03, 多重峰; C-4 上的 H 在化学位移 $\delta 6.78 ; \mathrm{C}-7$ 亚甲基由于酯基的影响出现在化学位移 $\delta 5.01$, 单峰; 环丙基结构中 C-9, C-10, C-11 上的 H 由于它们之间的相 互影响, 均裂分为多重峰, 化学位移分别在 $\delta 1.85 \sim$ $1.89,1.04 \sim 1.07,0.98 \sim 1.00$.

在 $3 \mathrm{~h}$ 的 IR 谱图中, 在 $1735 \mathrm{~cm}^{-1}$ 出现了酯羰基的 伸缩振动吸收峰, 而醌环上的羰基由于与双键共轭, 吸 收出现在低波数 $1656 \mathrm{~cm}^{-1}$; 环丙烷的 $\mathrm{C}-\mathrm{H}$ 伸缩振动 在 $3053 \mathrm{~cm}^{-1}$ 区域.

\section{3 结论}

本文设计、合成了小蠊醌的 20 个类似物 $\mathbf{2 a} \sim \mathbf{2} \mathbf{j}$ 和 $\mathbf{3 a} \sim 3 \mathbf{j}$, 除 2a, 3a, 2f, 3f 外, 其余化合物均未见文献报 道, 结构经过 ${ }^{1} \mathrm{H}$ NMR, IR 和 HRMS 分析确证. 为化合
物进一步的生测实验及构效关系研究奠定了基础.

\section{References}

[1] Fakoorziba, M. R.; Eghbal, F.; Hassanzadeh, J.; MoemenbellahFard, M. D. Ann. Trop. Med. Parasitol. 2010, 104, 521.

[2] Londres, M. I.; Sarinho, F. W.; Miranda, P. J.; Solé, D.; Sarinho, E. Clin. Lab. 2011, 57, 969.

[3] Wen, Z.; Scott, J. G. Pestic. Sci. 1997, 49, 367.

[4] Wu, D.; Scharf, M. E.; Neal, J. J.; Suiter, D. R.; Bennett, G. W. Pestic. Biochem. Phys. 1998, 61, 53.

[5] Wei, Y.; Appel, A.; Moar, W. J.; Liu, N. Pest. Manage. Sci. 2001, $57,1055$.

[6] Morrison, G.; Barile, J.; Macom, T. E. Pest Control Technol. 2004, $32(2), 62$.

[7] Wang, C. L.; Scharf, M. E.; Bennett, G. W. J. Econ. Entomol. 2004, 97, 2067.

[8] Nojima, S.; Schal, C.; Webster, F. C.; Santangelo, R. G.; Roelofs, W. L. Science 2005, 307, 1104.

[9] Liang, D.; Schal, C. Experientia 1993, 49, 324.

[10] Abed, D.; Tokro, P.; Farine, J. P.; Brossut, R. Chemoecology 1993, $4,46$.

[11] Kostikov, A. P.; Popik, V. V. J. Org. Chem. 2007, 72, 9190.

[12] Barbosa, L. C. A.; Alvarenga, E. S.; Demuner, A. J.; Virtuoso, L. S.; Silva, A. A. Chem. Biodiverity 2006, 3, 553.

[13] Williamson, D. A.; Bowler, B. E. Tetrahedron 1996, 52, 12357.

[14] Kumar, S. K.; Amador, M.; Hidalgo, M.; Bhat, S. V.; Khan, S. R. Bioorg. Med. Chem. 2005, 13, 2873. 